\title{
The canopy effect in AEM revisited: investigations using laser and radar altimetry
}

David Beamish $^{(a)}$ and Hanna Leväniemi ${ }^{(b)}$

(a) British Geological Survey, Keyworth, Nottingham, NG12 5GG, UK.

(b) Geological Survey of Finland, PO Box 96, FI-02151 Espoo, Finland.

\section{Corresponding author:}

David Beamish

British Geological Survey, Keyworth, Nottingham, NG12 5GG, UK

Email: dbe@bgs.ac.uk.

Tel: +44(0)1159363432

Fax: +44(0)115936 3261

Keywords:

Airborne geophysics, electromagnetic methods, radar, laser, altimetry

Near Surface Geophysics 


\section{Abstract}

This study considers a specific issue, often termed the canopy effect that relates to our ability to provide accurate conductivity models from airborne electromagnetic (AEM) data. The central issue is one of the correct determination of sensor height(s) above the ground surface (terrain clearance) to the appropriate accuracy. The present study uses the radar and laser systems installed on a fixed-wing AEM system to further investigate the effect. The canopy effect can arise due to a variety of elevated features below, and in the vicinity of, the flight line. The most obvious features are welldefined forest and copse zones together with domestic, commercial and agricultural buildings. Such features may cause the terrain clearance to be underestimated and this has the potential to introduce resistive artefacts and incorrect interface depths into conductivity models. Correct determination of terrain clearance is also important for the accurate processing of the other geophysical data sets acquired by airborne surveys. Radar and laser altimetry offer two very different physical measurements of height above ground. Airborne radars detect the range to the nearest reflecting object. They do this over a cone of influence that may have a radius (at the ground surface) of 55 m (assuming a survey height of $60 \mathrm{~m}$ ). Reflections from objects that are off-line are thus a distinct possibility. In direct contrast, a laser ranging device with low beam divergence provides a highly focussed measurement. Laser accuracies (typically < $2 \mathrm{~cm}$ ) are far greater than those of radars (typically $\sim 0.5 \mathrm{~m}$ ). In addition, the rapid sampling of laser ranging devices (e.g. up to $2 \mathrm{kHz}$ ) allows both real-time and post- 
processing algorithms to be applied to estimate the maximum range recorded across appropriate time/spatial windows. In our case a $2 \mathrm{kHz}$ (maximum) dual-pulse laser when sampled at $200 \mathrm{~Hz}$ provides a x50 oversampling in relation to the $4 \mathrm{~Hz}$ sampling of the EM components. Our studies from recent surveys indicate that the radar altimeter often provides significantly underestimated values of terrain clearance. Such estimates may be in error by up to 10 or $15 \mathrm{~m}$ across dense woodland. The issues of accuracy and reliability raised by the routine application of laser altimetry to AEM surveys and conductivity models are evaluated. It is demonstrated that it is now possible to obtain reliable laser estimates of true height above ground surface across:

(a) most forms of canopy encountered, (b) built structures of limited spatial scale and (c) bodies of water. 


\section{Introduction}

Airborne electromagnetic (AEM) surveying can employ frequency-domain or timedomain techniques. The frequency-domain systems, considered here, currently exist as towed-bird configurations comprising coil sets within a rigid sensor boom towed beneath a helicopter (HEM systems) and as fixed-wing (wing-tip sensor) mounted coil sets. The frequencies employed by the two systems are similar with the HEM configurations capable of recording to a higher frequency, in excess of $100 \mathrm{kHz}$. Transmitter-receiver separations are typically 8 to $9 \mathrm{~m}$ in modern HEM sensor birds while fixed-wing system separations can exceed $21 \mathrm{~m}$. These differences lead to potential differences in operational heights in terms of maintaining adequate signal/characteristics. A comparison of typical HEM and fixed-wing responses (coupling ratios) was presented by Beamish (2003). The provision of adequate signal/noise at survey sensor elevations above $50 \mathrm{~m}$ is a critical factor when flying regulations preclude surveying at lower flight altitudes. Broadly, in terms of general AEM surveying behaviour, we can anticipate coil sensor elevations in the range 30 to $60 \mathrm{~m}$, although exceptions are inevitable.

Altitude estimation in airborne systems may utilise a variety of sensors and procedures. These include differential or multi-receiver GPS (required as part of survey navigation and accurate survey data location). Radar and laser altimeter systems are the primary sensors used to determine the height of the sensors above ground surface. Multi-parameter geophysical systems may also include on-board barometric altimeters (recording height above mean sea level) and are typically used for STP (Standard Temperature and Pressure) airborne radiometric calculations. 
Accuracies and behaviours of these systems are considered later. The main focus of interest for the purpose of this study are the radar and laser altimeters used to determine sensor (i.e. the transmitter and receiver coil pairs) height above ground surface. The parameter is highly significant in the estimation of conductivity data and models derived from the observed coil-coil coupling ratios. A study using only radar altitude information was presented by Beamish (2002a).

Forward modelling of AEM responses allows the prediction of signal/noise performance of specific AEM systems and coil configurations. It is also possible to predict the accuracy required of the measurement of height above ground. Both measurement accuracies are related to the predicted, measured or assumed noise level of the coupling ratios. In general the highest frequency of each system determines the highest accuracy that should be achieved. In addition, the altitude accuracy requirement is more stringent for surveys performed over conductive environments and at low altitude. The sensitivity of coupling ratios to altitude is system specific and the investigations performed in this study are based on the AEM-05 fixed wing (Twin Otter) frequency-domain system and associated measurement systems described by Leväniemi et al. (2009). The system incorporates 4 dual vertical coplanar coils mounted on the wingtips and provides a bandwidth from $900 \mathrm{~Hz}$ to $25 \mathrm{kHz}$.

A simple comparison of typical radar and laser systems used in AEM surveys reveals the following. The typical and stated accuracy of a modern laser system is a few centimetres while that of a typical radar may be or the order of $0.5 \mathrm{~m}$.

This is demonstrated in Figure 1 which shows well-calibrated radar (Ralt) and laser (Lalt) altimeter data, sampled at $4 \mathrm{~Hz}$, obtained across a $1 \mathrm{~km}$ section of a lake. The 
survey aircraft undergoes a $4 \mathrm{~m}$ altitude undulation over the $1 \mathrm{~km}$ profile however the stated accuracies of the two devices appear to be observed. A second difference in the two types of altitude measurement lies in their physical beamwidths. A radar system, operating pulse modulation at a frequency of $\sim 4.3 \mathrm{GHz}$, may have a beamwidth defined by an angle of up to $68^{\circ}$. The system will measure the distance to the nearest reflecting object within a spatial scale defined by a 3D cone of influence as indicated schematically in Figure 2. Reflections from objects that are off-line are a distinct possibility, particularly as survey elevation increases. In direct contrast, a modern laser system data is capable of very rapid sampling with a very low beam dispersion (typically 1 to 2 mrad). At normal AEM survey altitudes, a laser system should provide a zone of influence that is approximately a vertical tube $<15 \mathrm{~cm}$ in diameter at the ground surface. This is indicated schematically in Figure 2. A final difference that stems from their physical beamwidths, is their differing abilities to provide accurate measurements of height above ground surface in the presence of elevated features. Features such as forest and copse zones together with domestic, commercial and agricultural buildings may cause either of the two systems to record an underestimated ground clearance and this is generally called the canopy effect (e.g. Fraser, 1978; Beamish, 2002a). As is demonstrated here, a conventional laser altimeter system in the presence of light canopy can provide penetration to the ground surface. Recent laser systems deployed on fixed-wing platforms and in EM birds have used a dual-pulse laser altimeter (Ahl and Winkler, 2006; Leväniemi et al., 2009). The AEM-05 system incorporated a Riegl dual pulse (meaning that it can differentiate the times of the first and last pulse it receives) laser in 2005. The device has a maximum pulse sampling rate of $2 \mathrm{kHz}$. The device is programmable so that for longer measurement times, single laser shots are averaged. The device then returns amplitude 
and quality factors of the reflection characteristics. The rapid sampling of laser ranging devices allows both real-time and post-processing algorithms to be applied to estimate the maximum range recorded across appropriate time/spatial windows. It is this extended ability that allows for an increased accuracy and uniformly reliable measurement of height above ground surface for all AEM surveys.

\section{Altitude estimation in airborne systems}

Although different airborne systems employ different altitude estimation configurations, a common set of modern survey navigation/altitude sensors includes one or more multi-channel differential GPS systems providing $\mathrm{x}, \mathrm{y}, \mathrm{z}$ location. In these systems the vertical (z) measurement provides height above a reference geoid (e.g. WGS-84). Radar and laser altimeters are used to measure height above ground surface and a barometric sensor to measure height above mean sea level.

The accuracy of the GPS vertical measurement is linked to the overall accuracy of the GPS installation and the processing that is carried out to reduce the error terms inherent in GPS location measurements. These are discussed in relation to the estimation of digital elevation models derived from airborne geophysical survey data by Richardson (2000) and Hauteneimi et al. (2005). Currently differential GPS involving an-board GPS receiver operated with a base station receiver, located in the survey area, is a common operational format. In broad terms the vertical GPS-z measurement is inherently less accurate than the GPS-x,y measurement. 
Differential GPS reduces the effects of these errors and provides improved accuracy to the order of several metres. Note that calculated horizontal positions are more accurate than associated ellipsoidal heights because of the influence of satellite geometry on vertical positions. Some of the errors listed above influence the vertical component much more than the horizontal component. A rule of thumb is that the ellipsoidal height accuracy is 1.5 times worse than the horizontal accuracy (Natural Resources Canada, 1995).

The differential GPS system operated as part of the AEM-05 system is well described by Hautaniemi et al. (2005). The system employs single frequency (L1) GPS+GLONASS constellations although the GLONASS network is now not fully operational. To improve the observed GPS+GLONASS data, a differential correction, using base station data, is applied to the observed coordinate data. In the differential correction, errors caused by ionospheric and tropospheric refraction, ephemeris and lock errors are significantly reduced. Coordinate transformation from WGS84 to local planar coordinate system is also undertaken. It is estimated that with single frequency GPS+GLONASS receivers in differential mode, and using appropriate commercial GPS software tools, we can measure the GPS-z reference height to a typical accuracy of about 1.5 metres (Hauteneimi et al., 2005).

Radar and laser altimeter systems are discussed in more detail later. A typical radar system may have an accuracy of about $0.6 \mathrm{~m}$ (at $30 \mathrm{~m}$ elevation) increasing to $\sim \mathrm{m}$ at elevations above $50 \mathrm{~m}$. A modern laser system is far more accurate and a typical 
accuracy is a few (e.g. 2 to 3) centimetres. On-board barometric altimeters (recording height above mean sea level), are typically used for STP (Standard Temperature and Pressure) airborne radiometric calculations. To increase reliability and accuracy for height estimation (e.g. for EM applications) they require simultaneous base station or radio-link control tower information to accommodate inherent atmospheric weather variations. Evaluations of the accuracies of these systems have been undertaken by Aviation Authorities (e.g. the Federal Aviation Authority) using simulation models. Typically, sustained accuracies of better than several metres cannot be expected.

In broad terms the two main, sub-metre measurements of sensor elevation above ground currently available are those provided by radar and laser systems. It is acknowledged that this situation may change as multi-frequency, multi-receiver GPS and real-time kinematic networks and procedures continue to evolve. Despite the theoretical accuracies described above, the two main measurements are known to suffer from high inaccuracies ( $>50 \%$ ) when attempting to record height above ground level across dense canopy and elevated structures. The abilities of these systems to provide accurate and reliable sensor elevations for airborne EM applications is the main issue investigated in this paper.

Canopy effects, due to elevated features, result in underestimated radar and laser altimeter readings of height above ground. A second class of effect, termed the paddock effect, has been noted by Richardson (2000) and is further described by Brodie and Lane (2003). The paddock (meaning a ploughed portion of land) effect, as currently defined, results in overestimated radar altimeter readings only. It is suggested that a possible reason for the observed paddock effect is that surface 
roughness of the same order as the wavelength of the radar $(=7 \mathrm{~cm}$ for a $4.3 \mathrm{GHz}$ radar) influences the shape and timing of the radar reflections. A reduction in amplitude, or a shift in the timing, appears to result in an overestimated estimate of time-travel and hence altitude. Richardson (2000) and Brodie and Lane (2003) provide examples in which the radar altitude is overestimated by 3 to $5 \mathrm{~m}$ in these circumstances.

The inter-calibration of radar and laser altitude estimates is best performed, or verified, over bodies of still water such as lakes. When such estimates are compared on an individual survey basis, a typical pattern emerges as shown in Figure 3. The radar (Ralt) and laser (Lalt) elevation estimates shown come from a small detailed survey (1700 line-km) that employed a line-spacing of $75 \mathrm{~m}$. The survey area in southern Finland is predominantly rural (fields, forests and lakes). The procedure used to obtain the laser estimates (the Lmax algorithm) is described later. The data, sampled at $4 \mathrm{~Hz}$, comprise $\sim 150,000$ points and the nominal survey elevation is $30 \mathrm{~m}$. The distinct bias to significantly positive values of the difference Lalt - Ralt is evident. Some $79 \%$ of the altitude estimates provide values with Lalt minus Ralt greater than, or equal to, zero. The smaller amount of apparently overestimated Ralt estimates (i.e. Lalt $<$ Ralt) are largely confined to an amplitude range of $<4 \mathrm{~m}$. A detailed investigation was carried out in relation to possible associations between areas of cultivated land (as defined on maps) and the occurrence of the condition Lalt $<$ Ralt. No persistent associations, that would have supported the paddock effect, were found. 


\section{Altitude dependence of AEM coupling ratios}

The sensitivity of coupling ratios to altitude is system specific. Here we consider only the AEM-05 system. The behaviour of coupling ratios with altitude is an important element in determining the accuracy required in the associated estimate of altitude. The highest frequency of each system determines the highest accuracy required. The altitude accuracy requirement is most stringent for surveys performed in conductive environments and at low altitude.

As an example, we consider the AEM-05 system and the highest frequency of 24,510 Hz. Using a survey altitude of $30 \mathrm{~m}$ we calculate the response for a series of half space conductivities for sensor elevations that may be underestimated by up to $10 \mathrm{~cm}$ (i.e. an elevation range from 29.90 to $30 \mathrm{~m}$ ). The response used is the modulus of the complex (real and imaginary) coupling ratios. The results for a resistive half space (1000 ohm.m) and a moderately conducting half space (10 ohm.m) are shown in Figure 4. The coupling ratios for an altitude of $30 \mathrm{~m}$ are 2258 ppm (1000 ohm.m) and 23,984 ppm (10 ohm.m) and the results are shown normalised to these reference response values.

The results obtained for the 1000 ohm.m half-space are clearly insensitive to errors in altitude. The variation over the $10 \mathrm{~cm}$ range is less than $10 \mathrm{ppm}$ which is within the typical noise envelope of acquired survey data. This implies that a $10 \mathrm{~cm}$ altitude difference ascribed to the altitude would not unduly influence the models obtained across entirely resistive terrains. In the more conductive $10 \mathrm{ohm} . \mathrm{m}$ environment, response differences extend to $186 \mathrm{ppm}$ indicating that a $10 \mathrm{~cm}$ error in altitude at a 
nominal survey elevation of $30 \mathrm{~m}$, would be significant (well above typical acquired noise figures at this frequency). If we were to adopt a precise noise level of, say 60 ppm, then the required accuracy of the altitude estimate is predicted to be $\sim 3 \mathrm{~cm}$. This is clearly beyond the accuracy available from typical radar systems and can only be achieved with a laser ranging device. In practice, since the sensor is moving at $\sim 15 \mathrm{~m}$ per second, the centimetre accuracy would only be truly meaningful in the case of a perfectly flat surface. The influence of altitude error decreases with decreasing frequency. The equivalent response at a lower frequency of $3005 \mathrm{~Hz}$ is also shown in Figure 4.

As an indication of the differential effects of altitude with frequency it can be noted that, for the $10 \mathrm{ohm} . \mathrm{m}$ half space model and an elevation of $56 \mathrm{~m}$, an elevation difference of $10 \mathrm{~cm}$ produces response differences of only $24 \mathrm{ppm}$. Since AEM surveys may be conducted at a variety of nominal elevations, it is the lowest elevation that determines the required accuracy of the height above ground measurement.

When interpreting data from a multi-frequency system, the different sensitivities of the coupling ratios at each frequency produce a different error at each frequency if an error in the altitude assignment occurs. The errors in coupling ratios due to an incorrectly assigned altitude increase with both conductivity and frequency. Thus if a conductivity model is determined from multi-frequency data with an underestimated altitude, the model is progressively biased (to a progressively more resistive estimate) with increasing frequency. This has the net result of providing overestimated 
resistivities in the upper sections of the model. The effect on two forms of conductivity model is now described.

\section{Influence of altitude errors on 1D conductivity models}

It should first be noted that the standard single frequency Fraser half-space transformation (Fraser, 1978), when applied using both in phase and quadrature coupling ratios, is effective in returning resistivity estimates unbiased by errors in the altitude estimate (Beard, 2000). Beamish (2002b) suggested the Fraser pseudo-layer procedure resulted in conservative estimates of conductivity when compared to estimates obtained by equivalent iterative, minimisation procedures.

In order to distinguish vertical resistivity variations and obtain a formal measure of model validity one can use a multi-layer 1-D inversion (Beard, 2000; Constable et al., 1987; Fitterman and Deszcz-Pan, 1998; Paterson and Redford, 1986; Sengpiel and Siemon, 1998). The models returned by such inversions are non-unique because they typically solve an underdetermined problem. They therefore employ additional constraints to stabilise the problem, such as the Marquardt-Levenberg regularisation or smoothness constraints. Smoothness constraints can be applied in the vertical or horizontal (lateral) directions.

Multi-layer iterative minimisation inversion methods can be used to provide both uniform half space models (Beamish, 2002b) and models containing few or many layers (Tølbøll and Christensen, 2006). Here we consider how altitude errors 
influence both few and many layer models when altitude is assumed to be known (and accurate) in an inversion procedure.

The synthetic model comprises 2 conductive layers $(100 \mathrm{mS} / \mathrm{m})$ in a resistive (1 $\mathrm{mS} / \mathrm{m}$ ) host. The first conductive layer has a thickness of $5 \mathrm{~m}$ and is located between depths of 10 and $15 \mathrm{~m}$. The second conductive layer has a thickness of $20 \mathrm{~m}$ and is located between depths of 60 and $80 \mathrm{~m}$. The model has five layers including the underlying half space. The model is used to calculate synthetic responses for the AEM-05 system at a fixed elevation. In this study, an elevation of $50 \mathrm{~m}$ is used. Incremental errors to the correct altitude are then introduced to allow an assessment of the model distortions introduced by using an incorrect altitude.

The altitude-error effect on a few layer model assessment is considered first. The inversion method used is the constrained conjugate gradient inversion described by Tartaras and Beamish (2006). Although developed for the application of lateral constraints to model calculations, the method is inherently stable and does not require regularisation even when lateral constraints are not used. In the case of the discrete altitude-error behaviour, lateral constraints were not employed.

When dealing with survey data, the actual (or most appropriate) number of layers must be evaluated. In the case of the synthetic data, the number of layers is known precisely. The synthetic model was used to generate the 4 frequency fixed-wing response assuming a survey elevation of $50 \mathrm{~m}$. Gaussian random errors at the $2.5 \%$ level were then added to the data. A series of altitude errors of between -15 m (i.e. underestimated elevations) and +5 m (i.e. overestimated values) were then introduced 
to the data in $1 \mathrm{~m}$ increments. The results of inverting the data set are summarised in Figure 5. The model obtained using the correct altitude $(50 \mathrm{~m})$ is arrowed. All models across the range of altitude errors detect the 2 conductive layers within the resistive host. In broad terms the thickness of the upper conductor is approximately correct but the conductivity is underestimated. The latter result is connected with the limited thickness of the conductor rather than any error in altitude. The conductivity of the lower conductor is approximately correct but the thickness is persistently overestimated. The inversions return chi-square misfits (Constable et al., 1987) of 8 for all the models when $2.5 \%$ errors are assigned. Since the expectation of the data misfit is 8 , all the models are equally well-fitted. In this case, a ch-square misfit of 8 corresponds to an rms misfit of unity.

The effect of underestimated altitude errors on the few layer model is to artificially increase the depth estimate of the two conductive layers (for this model). A reciprocal situation occurs when altitude is overestimated and the depth to both conductive layers is underestimated. If we consider that altitude may be underestimated by $15 \mathrm{~m}$, then the depth of near surface conductive features may be overestimated by $\sim 15 \mathrm{~m}$ and the depth to deeper conductive features may be overestimated by more than $20 \mathrm{~m}$.

The corresponding behaviour in the case of many layer inversion models is now considered. An Occam (Constable et al., 1987) multi-layer procedure offering regularised, smooth models in the vertical direction is used (e.g. Farquharson et al., 2003). The advantage of the procedure is that there is no requirement to set the number of conductivity interfaces (layers) within the model. The models produced however cannot detect interfaces; neither can they recover 'true' layer conductivity 
values. The inversion model is essentially a smooth representation of the most likely 1D conductivity distribution. The inversion starting model used a uniform half space divided into 35 layers above a half space. For the synthetic model data, a standard error of $2.5 \%$ was assumed.

The results of inverting the data set are summarised in Figure 6. The model obtained using the correct altitude $(50 \mathrm{~m})$ is arrowed. All models across the range of altitude errors detect the 2 conductive layers within the resistive host as 2 local maxima in the conductivity profile. In the vicinity of the correct altitude, both conductivities are clearly underestimated. The depth to the near surface conductor displays a similar pattern to that observed in the few layer case. As the error in the underestimated altitude increases, the Occam minimisation attempts to fit the incorrect data (incorrect by virtue of the altitude assigned) by introducing more extreme (oscillatory) model behaviour. Again, the inversions return chi-square misfits of 8 for all the models when $2.5 \%$ errors are assigned.

Many of the published formal AEM inversion strategies do not appear to require the use of an equivalent pseudolayer (a fixed high resistivity at-surface layer of variable thickness) to accommodate errors in altitude. It is theoretically possible to include sensor altitude as an unknown parameter in an inversion strategy (e.g. Hodges, 2003). This has the general net effect of increasing the number of unknowns thereby increasing the parameter resolution issues within an already underdetermined problem. An alternative procedure in few layer inversion strategies was proposed by Beamish (2002b) and reiterated by Tølbøll and Christensen (2006). The air-layer technique introduces a high resistivity (equivalent to air) upper layer to represent 
sensor altitude above ground surface. The layer thickness is free to vary but the resistivity (e.g. 100,000 ohm.m) is highly constrained or fixed. The air-layer technique is more difficult to implement within a many layer, regularised inversion procedure since the first or second vertical derivative of the conductivity profile is constrained to be a smooth function.

Given the difficulties arising in the case of providing an accurate and reliable estimate of sensor height above ground, it would be preferable to investigate the degree to which the estimate can be improved. As already stated, it is likely that only laser altimetry can provide the centimetre accuracy required. The behaviour of both radar and laser systems is now investigated.

\section{Radar and Laser altimeter systems}

There are, of course, a variety of radar and laser altimeter systems employed in AEM systems. A typical and common radar installation manufactured by Honeywell/Sperry or by Collins operates at a frequency of around $4.3 \mathrm{GHz}$ (short pulse modulation). The accuracy of these devices is usually quoted as being the greater of two quantities i.e. an absolute figure of about $0.6 \mathrm{~m}$ or $2 \%$ of the elevation (at low survey altitudes). There is a general indication that even with spatial filtering of oversampled data, an accuracy level of better than $1 \mathrm{~m}$ may not be achieved with radar altimeters (Brodie and Lane, 2003). A second important parameter in radar altimeters is the beamwidth. Normally this is defined as the angle between two directions in a plane containing the maximum of the beam (i.e. the vertical plane in this case) in which the radiation 
intensity is half the maximum value of the beam or $-3 \mathrm{~dB}$. The radar altimeter effectively measures the distance to the nearest reflecting object within its beamwidth. A typical beamwidth figure appropriate to Honeywell/Sperry and Collins radar systems is 50 degrees, although mean estimates may vary between values of 45 and 68 degrees.

Modern laser altimeter systems of the type employed in AEM systems (e.g. manufactured by Optech and Riegl) have a typical pulse repetition rate of up $2 \mathrm{kHz}$. Laser systems deployed in bird systems appear to sample the laser data at the fastest geophysical channel rate e.g. $10 \mathrm{~Hz}$. Specifications of such systems indicate that the laser altimeter measures the distance from the EM bird to the ground, except in areas of dense tree cover. The disturbances to laser EM bird altimeter data caused by trees and buildings are noted by Tølbøll and Christensen (2006) and Siemon (2009).

More recent laser systems deployed on EM birds have used a dual-pulse laser altimeter (Leväniemi et al., 2009, Ahl and Winkler, 2006). The AEM-05 system, considered here, incorporated a Riegl dual pulse (meaning first and last pulse) laser in 2005. The device has a maximum pulse sampling rate of $2 \mathrm{kHz}$. The device is programmable so that for longer measurement times, single laser shots are averaged. The device then returns amplitude and quality factors of the reflection characteristics. A last target detection capability of multiple (or obscured) targets is also provided. In theory, the control provided should allow the laser to be 'tuned' for routine measurement of distance to the ground surface through obscuring reflectors such as vegetation and canopy (although not through solid structures such as buildings). The 
procedures applied to the processing of such laser data are now described and a detailed case study is presented.

\section{The Lmax algorithm}

In practice, by operating the laser at $200 \mathrm{~Hz}$, an oversampling of x50 is achieved in relation to the standard $4 \mathrm{~Hz}$ sampling of the EM components. The averaging is useful for accurate detection of badly reflecting targets or for targets with changing reflection characteristics during measurement (e.g. a water surface). At a typical flying speed of $60 \mathrm{~m} / \mathrm{s}$, the raw $2 \mathrm{kHz}$ sampling equates to a shot every $3 \mathrm{~cm}$. Averaging over 10 points to provide a $200 \mathrm{~Hz}$ estimate equates to a distance of $30 \mathrm{~cm}$.

The laser altimeter data are recorded as a separate data stream at $200 \mathrm{~Hz}$.

The 50 point redundancy in the sampling then allows a post-processing algorithm, based on independent windows of 50 samples, to obtain an accurate (although necessarily spatially-averaged) estimate of height above ground surface for the EM data channels (i.e. resampled to $4 \mathrm{~Hz}$ ). The simplest procedure is to determine the maximum value of the laser data across the 50-point data window. This procedure is referred to here as an Lmax estimate. In most cases this provides an effective measure of true height above ground surface through vegetation and most types of canopy we have encountered. In extreme cases of high reflectance (sun on water), all individual data point may be null returns and, in this case, the final estimate is a zero value.

It should also be noted that the stated accuracy of measurement of such systems is 2.5 $\mathrm{cm}$ and this should be readily achieved when averaging raw $2 \mathrm{kHz}$ data across 
reduced time intervals such as $200 \mathrm{~Hz}$. In addition the most important feature of the laser measurement is that it is a beam with a limited amount of divergence. The stated beam divergence figures for the AEM-05 device indicate that the beam diameter on the ground will be less than $15 \mathrm{~cm}$ for most survey flight elevations.

The behaviour of the $200 \mathrm{~Hz}$ laser data in relation to its resampled form and the radar data is best illustrated using a detailed example above a building. The use of a welldefined structure, rather than canopy, provides a more robust example with clean edge effects. Figure 7a shows a downward-looking image from the AEM-05 video system above a single structure within an industrial estate in the UK. Survey altitude above ground is about $66 \mathrm{~m}$ and the building is about $40 \mathrm{~m}$ wide (edges $\mathrm{A}$ and B) and $5 \mathrm{~m}$ high. The flat roof of the building has a series of 4 ventilation structures, each about 1 m high. The flight path traverses the centre of the image as shown by the dash line.

Figure 7b shows the $200 \mathrm{~Hz}$ raw and $4 \mathrm{~Hz}$ resampled Lmax laser data, together with the radar altitude recorded across the structure. The total elevation range shown is 11 m. The $200 \mathrm{~Hz}$ laser data defines reflections from the main edges of the building (A and B) together with the ventilation structures. The general decreasing trend in elevation is due to a small $(<2 \mathrm{~m})$ aircraft descent along this portion of the flight line. The variation is recorded by the GPS-z (height above geoid) data shown (a value of $73 \mathrm{~m}$ was subtracted to contain the data within the scale of the graph). Across the central structure, both radar and the $200 \mathrm{~Hz}$ laser data provide underestimated values of height above ground level. The less accurate behaviour of the radar altimeter (i.e. it is perturbed by two structures) can also be noted. On the open ground to the north of 
the structure, radar and laser data return correct height above ground some $20 \mathrm{~m}$ from the northern edge of the building.

The Lmax $4 \mathrm{~Hz}$ laser data is shown by the line with open square symbols. Two 50point data windows centred on estimation points 1 and 2 are indicated. Using the simple Lmax algorithm these points would have been underestimated due to width of this large structure. Most structures encountered at low survey elevations are, in fact, single structures such as rural houses and farms. The simple Lmax algorithm is able to obtain accurate height above ground across the majority of these structures of limited spatial extent. Since the perimeters of many conurbations in the UK now contain industrial units of similar scale to that used in this example, a revised Lmax algorithm has also been evaluated here. In this case, an expanding window technique (e.g. using successive expansions of 50, 100, 200 data points), allows the adjacent spatial consistency of different estimates to be compared. Clearly many aspects of the basic Lmax algorithm can be developed to suite different circumstances and requirements.

The extent to which laser systems are capable of providing accurate and reliable estimates of height above ground surface for AEM applications are now considered in a case study.

\section{Survey case study}

The case study example considers a portion of single survey line acquired across the edge of a lake, then across a dense canopy and then across a single isolated building. 
The features are included within a profile length of some $660 \mathrm{~m}$. Selected flight video images (Figure 8) show a lake in sunlight (labelled A), a central deciduous wooded area (labelled B) and an isolated building (labelled C). Figure 9 then shows a comparison of radar altitude (RALT, $4 \mathrm{~Hz}$ ) and laser altitude $(200 \mathrm{~Hz}$, raw and $4 \mathrm{~Hz}$, Lmax resampled) over a time interval of 11 seconds. Assuming a survey speed of 60 $\mathrm{m} / \mathrm{s}$, the corresponding distance is $660 \mathrm{~m}$.

Over the lake (A), the $200 \mathrm{~Hz}$ laser data suffers a series of zero amplitude (null) returns that are classed as zero altitude. The radar altitude returns are stable across what is assumed to be a flat body of water. Across the wooded area (B), the $200 \mathrm{~Hz}$ laser data show a complex sequence of returns (altitudes between 46 and $68 \mathrm{~m}$ ) that may be interpreted as true height above ground surface (e.g. largest altitudes, full penetration through the canopy,) and the height of the upper canopy (smallest altitudes). Between these 2 limits, we assume partial penetration of the canopy occurs. The radar, with a much lower (averaging) spatial resolution, shows a characteristic smooth decrease from the woodland edges (less dense foliage) through to the centre of the woodland (foliage most dense, and taller trees). The laser clearly identifies the isolated building (C), a sports pavilion, together with the slope of its pitched roof.

The resampled $4 \mathrm{~Hz}$ laser for the example is shown in Figure 9 by the blue trace. Over the body of water we obtain correspondence between the radar and laser estimates. Across the forested area, a realistic upper bound to the raw $200 \mathrm{~Hz}$ laser data is obtained. This upper trace follows the same trend observed in the GPS height above geoid (shown as the uppermost trace). The depression observed in the radar altitude across zone B is interpreted as reflecting the height of the canopy. The results 
indicate that errors in the radar estimate of height above ground extend to $11 \mathrm{~m}$, in this example.

The effect of the underestimated radar altitude on conductivity models for these data has been investigated by comparing the results obtained using Ralt and Lmax as the known altitude parameter in an inversion procedure. Here we consider the two cases of few and many layer inversion procedures, as previously described. The many layer Occam procedure is considered initially as it provides an indication of the most appropriate number of layers for use in subsequent few layer inversions. As in the previous synthetic data study the inversion starting model used a uniform half space divided into 35 layers above a half space. A simple and uniform standard error of 5\% was assumed as the error model for the data.

The conductivity models obtained are compared in Figure 10a (Ralt) and 10b (Lmax). The lateral extent of the central canopy effect as defined by the Lmax and Ralt altitude differences is indicated by arrows. The zone of maximum canopy effect ( $\sim 12$ $\mathrm{m}$ ) is indicated by the shaded rectangles. Both models reveal 2 conductive layers, the first a shallow thin zone followed by a deeper, more conductive and thicker zone. The geological context is a Quaternary sequence with the higher conductivities being ascribed to clay content. The perturbation to the model obtained using Ralt (Figure 10a) is similar to that observed in the synthetic model study. In the case of the survey data, the canopy effect is progressive and leads to a depression in both shallow and deeper conductive zones. A further effect is to produce a highly resistive zone $(<0.5$ $\mathrm{mS} / \mathrm{m}$, as indicated) in the at- and near-surface zone of maximum height of canopy. 
Assuming a 5\% error model, the chi-square misfits range from the expectation level of 8 to a maximum of 13 (Ralt) and 14 (Lalt).

The smooth Occam models essentially map gradients in the vertical conductivity profiles. As can be seen in Figure 10, there are large apparent variations in the location of the maxima in conductivity, particularly in the deeper conductive zone, across the profile. A few layer inversion with lateral constraints can also be applied to the data, to reduce the point-to-point variability in the conductivity models and to better demonstrate the canopy effect. The inversion method used is the constrained conjugate gradient inversion described by Tartaras and Beamish (2006). Following the previous Occam analysis, a 5 layer model configuration was assumed appropriate. The conductivity models obtained are compared in Figure 11a (using Ralt) and Figure11b (using Lmax). The canopy parameters are again indicated by arrows and the grey rectangles. In the laterally-constrained few layer models, the canopy effect is much clearer. In the Ralt inversion (Figure 11a) the perturbation in the depth to the top of each conductor follows the general behaviour of Ralt observed in Figure 9. The thickness of each layer remains uniform across the profile however the behaviour observed is linked to the fact that the starting model used is matched to that determined by the preceding Occam analysis. Again, the chi-square misfits are found to be within 2 standard deviations of the expectation values for both sets of inversion results. The inversions thus appear to be fitting both data sets equally well.

\section{Laser altimeter behaviour across bodies of water}


One of the aspects concerning the reliability of laser altitude measurements is their ability to provide valid measurements across bodies of water undergoing a high light reflectance due to the presence and angle of the sun. The water bodies may be inland lakes, rivers, estuaries or the sea. The previous survey case study provided a small example of the behaviour observed. The high reflectance condition may also occur over snow/ice covered land or sea. The degree to which this condition is encountered is obviously survey specific. For each survey undertaken it is possible to investigate the Lmax data set for zero/null returns (i.e. no reflections detected across a $3 \mathrm{~m}$ spatial window). When such analyses are carried out in conjunction with topographic maps it is possible to establish the locations of the zero returns. Using the flight-path video images it is also possible to establish the qualitative reflectance characteristics at the locations of the zero returns. Examination of large quantities of our survey data reveals that the almost exclusive majority of zero returns are indeed connected with water bodies and the presence, strength and angle of the sun. It should be noted that some surveys over areas containing lakes have produced no zero returns and this is simply related to the prevailing weather. Low sun angles (e.g. early morning) can produce localised zones of zero returns. Figure 12 is an example, taken from part of a detailed survey area in southern Finland. The rectangle shown on a topographic background map was surveyed using N-S flight lines with a separation of $75 \mathrm{~m}$. The symbols are posted at locations along each flight line which provide null returns using the standard Lmax algorithm. The background topographic map identifies the locations of all the null laser returns with lakes (shown in blue). In this example, the null sequences along each flight line do not involve large numbers of estimates and an interpolation through each sequence might be considered appropriate. 
High angle, strong sun reflections over extensive water bodies can provide spatially extensive null returns in laser altimeter data. In these cases it is necessary to assume that the water body is flat and that the Ralt measurement can provide an adequate measurement of height above the water surface. The method adopted in such circumstances is simply to replace the laser height estimate (a null) with that obtained by the radar altimeter.

\section{Summary and conclusions}

The accurate and reliable estimation of height above ground (sensor elevation) contributes to the successful determination of conductivity models from AEM data sets. Both radar and laser altimeters are currently in routine use in modern AEM systems. The accuracy in sensor elevation required by a given AEM system is determined by the highest frequency employed and the associated noise level of the EM response at that frequency. Required sensor elevation accuracies increase at lower flight elevations and with increasing conductivity. Most modern AEM systems require the centimetre accuracy only available with laser ranging devices.

The canopy effect has the potential to provide significant (up to 50\%) errors in the measurement of sensor elevation when AEM surveys are conducted at low altitude. When canopy/structures are encountered, the differences in beamwidths of the two devices can provide different estimates of sensor elevation. When an underestimated 
altitude is used to derive a few or many layer conductivity model from multifrequency data, the net effect is to introduce near-surface resistive artefacts into the model. Depths to deeper conductive zones may also be significantly overestimated. Examples of the behaviour of conductivity models due to incorrect altitude assignments have been provided here for both synthetic and survey data. Further discussions of the modelling and interpretation complexity introduced by the unreliability of sensor elevation estimates can be found in Tølbøll and Christensen (2006) and Siemon et al. (2009).

Given the spatial complexity and persistence of canopy/structure effects in AEM data sets, it is worth developing a more rigorous approach to the accurate and reliable estimation of sensor elevation. It has been demonstrated that modern laser ranging devices are capable of accurate $(\sim 2 \mathrm{~cm})$ and rapid (e.g. up to $2 \mathrm{kHz})$ sampling. These characteristics allow both real-time and post-processing algorithms to be developed. The simplest procedure, here referred to as the Lmax algorithm, is to estimate the maximum range recorded across appropriate time/spatial windows. These subset estimates are found to provide reliable estimates of height above ground surface at spatial scales of the order of a few tens of centimetres.

The main benefit of such measurements and procedures is in relation to sensor elevation estimation in AEM surveys across zones of canopy and elevated structures. These are prevalent, for instance, in all surveys conducted in the UK. As the case study examples presented here demonstrate, it is now possible to obtain reliable laser estimates of sensor elevation across: (a) most forms of canopy (including vegetation) encountered, (b) built structures of limited spatial scale and (c) bodies of water. In 
cases where the sun angle on large bodies of water produces extensive null laser returns, it is necessary to replace laser estimates with those from the radar altimeter.

The laser measurement has a low beamwidth dispersion. This makes it amenable to simple correction for aircraft/bird orientation effects. Fitterman and Yin (2004) discuss the many aspects of bird manoeuvre distortion on frequency-domain helicopter-borne responses. Devices, such as GPS gyroscopes, that measure pitch, heading and roll are now common and the cosine correction of angles formed by pitch and roll allows a straightforward correction of the laser estimation of height above ground.

Finally it is worth noting that, following the technical discussions of radar and laser altimeters, the estimate of radar height is essentially a form of Digital Terrain Model (a DTM, i.e. it contains the effects of vegetation and elevated structures). When processed (e.g. the Lmax algorithm) laser altimeter data is also available, the height estimate conforms to that of a Digital Surface Model (a DSM, i.e. a bare-earth model devoid of surface effects). In certain cases these data sets may be available at much higher resolution than that obtained by an AEM survey. Where they are not available, the joint availability provides a potentially useful additional topographic mapping service.

\section{Acknowledgements}


This report is published with the permission of the Executive Director, British

Geological Survey (NERC) and the Programme Manager of the Geological Survey of Finland (GTK).

\section{References}

Ahl A. and Winkler, E. 2006. New AEM hardware developments at the Geological Survey of Austria. AEM Workshop Hannover, 2006. Airborne EM - Recent Activities and Future Goals. Extended Abstracts, p. 15.

Beamish D. 2002a. The canopy effect in airborne EM. Geophysics 67, 1720-1728.

Beamish D. 2002b. An assessment of inversion methods for AEM data applied to environmental studies. Journal of Applied Geophysics 51, 75-96.

Beamish D. 2003. Airborne EM footprints. Geophysical Prospecting 51, 49-60.

Beard L.P. 2000, Comparison of methods for estimating earth resistivity from airborne electromagnetic measurements. Journal of Applied Geophysics 45, 239-259. 
Brody R. and Lane, R. 2003. The importance of accurate altimetry in AEM surveys for land management. ASEG 16th Geophysical Conference and Exhibition, Adelaide, Extended Abstracts.

Constable S.C., Parker, R.L., and Constable C.G. 1987. Occam’s inversion: a practical algorithm for generating smooth models from electromagnetic sounding data. Geophysics 52, 289-300.

Farquharson C.G., Oldenburg D.W. and Routh P.S. 2003. Simultaneous 1D inversion of loop-loop electromagnetic data for magnetic susceptibility and electrical conductivity. Geophysics 68, 1857-1869.

Fitterman D.V. and Deszcz-Pan M. 1998, Helicopter EM mapping of saltwater intrusion in Everglades National Park. Exploration Geophysics 29, 240-243.

Fitterman D.V. and Yin, C. 2004. Effect of bird manoeuvre on frequency-domain helicopter EM response. Geophysics 69, 1203-1215.

Fraser D.C. 1978. Resistivity mapping with an airborne multicoil electromagnetic system. Geophysics 43, 144-172.

Hautaniemi, H., Kurimo, M., Multala, J., Leväniemi, H. and Vironmäki, J., 2005. The ‘three in one’ aerogeophysical concept of GTK in 2004. Geological Survey of Finland, Special paper 39, 21-74. 
Hodges, G., 2003. Practical inversions for helicopter electromagnetic data. 15th Annual Symposium on the Application of Geophysics to Environmental and Engineering Problems (SAGEEP), Proceedings, 45-58.

Leväniemi H, Beamish D., Hautaniemi H., Kurimo M. Suppala I., Vironmäki J., Cuss R.J., Lahti M. and Tartaras E. 2009. The JAC airborne EM system AEM-05. Journal of Applied Geophysics, 67, 219-233.

Natural Resources Canada, 1995. GPS Positioning Guide (third edition).

Paterson N. and Redford S.W. 1986. Inversion of airborne electromagnetic data for overburden mapping and groundwater exploration. In: Airborne Resistivity Mapping, Geological Survey of Canada, (Ed. G.J. Palacky), Paper 86-22, 39-48.

Richardson L.M. 2000. Errors in digital elevation models derived from airborne geophysical survey data. Australian Geological Survey Organisation, Record 2000/37.

Sengpiel K.P. and Siemon B. 1998, Examples of 1-D inversion of multifrequency HEM data from 3-D resistivity distribution. Exploration Geophysics 29, 133-141.

Siemon B. 2009. Levelling of helicopter-borne frequency-domain electromagnetic data. Journal of Applied Geophysics, 67, 206-218. 
Siemon B., Auken E., and Christiansen A.V. 2009. Laterally constrained inversion of helicopter-borne frequency-domain electromagnetic data. Journal of Applied Geophysics, 67, 259-268.

Tartaras E. and Beamish D. 2006. Laterally constrained inversion of fixed-wing frequency domain AEM data. Proceedings $12^{\text {th }}$ EAGE Near-Surface Conference 2006, Helsinki, Extended Abstracts B019.

Tølbøll R.J. and Christensen N.B. 2006. Robust 1D inversion and analysis of helicopter electromagnetic (HEM) data. Geophysics 71, 53-62.

\section{Figure Captions}

Figure 1. Example of Radar (Ralt) and Laser (Lalt) altimeter data recorded over a 1 $\mathrm{km}$ section of a lake. The sampling interval is $4 \mathrm{~Hz}$. The aircraft height undulates by 4 $\mathrm{m}$ across the $1 \mathrm{~km}$ profile.

Figure 2. Schematic representation of beamwidths associated with (a) a laser altimeter and (b) a radar altimeter.

Figure 3. Example of typical behaviour observed between Radar altitude (Ralt) and Laser (Lalt) altimeter data in a largely rural survey area. The Lalt data have been 
processed by the Lmax algorithm described in the text. The data sampling interval is 4 $\mathrm{Hz}$ and the plot contains $\sim 150,000$ data points although the plot has been restricted to a maximum height of $60 \mathrm{~m}$. The double-arrowed line indicates a scale of $20 \mathrm{~m}$.

Figure 4. Altitude dependence of coupling ratios across an elevation range of $10 \mathrm{~cm}$. Responses are relative and normalised to those at an altitude of $30 \mathrm{~m}$. The highest frequency of the AEM-05 system $(24510 \mathrm{~Hz})$ is shown for 2 half-space resistivity values (10 and 1000 ohm.m). The response at a lower frequency of $3005 \mathrm{~Hz}$ for a 10 ohm.m half-space is also shown for reference.

Figure 5. 1D inversion of synthetic model results illustrating the effect of incorrect altitude assignment on the detection of two conductive zones. The number of layers (4 above a half-space) is assumed known and a few layer inversion is used. The data are correct at an altitude of $50 \mathrm{~m}$. The results for a series of altitude errors between $-15 \mathrm{~m}$ (underestimated) and $+5 \mathrm{~m}$ (overestimated) are shown. The depths/thicknesses of the 2 conductive zones $(100 \mathrm{mS} / \mathrm{m})$ are indicated by dash lines.

Figure 6. 1D inversion of synthetic model results illustrating the effect of incorrect altitude assignment on the detection of two conductive zones. The number of layers is assumed unknown and a multi-layer smooth (Occam) inversion is used. The data are correct at an altitude of $50 \mathrm{~m}$. The results for a series of altitude errors between -15 m (underestimated) and $+5 \mathrm{~m}$ (overestimated) are shown. The depths/thicknesses of the 2 conductive zones $(100 \mathrm{mS} / \mathrm{m})$ are indicated by dash lines. 
Figure 7a. Image obtained from downward-looking video above an industrial unit. The flight path is shown by the horizontal dash line. The distance across the edges of the unit $(\mathrm{A}, \mathrm{B})$ is $40 \mathrm{~m}$. The survey elevation is $\sim 66 \mathrm{~m}$.

Figure 7b. Altitude estimates obtained across the industrial unit shown in Figure 7a. The main building is indicated by the arrowed line A,B, a second building is also indicated. The four estimates of altitude shown are Radar (Ralt at $4 \mathrm{~Hz}$ ), raw Laser at $200 \mathrm{~Hz}$, Lmax processed Laser at $4 \mathrm{~Hz}$. The height above geoid data (GPS-z) has had a constant value removed to allow inclusion in the plot.

Figure 8. Four images obtained from the downward-looking video across the case study flight line. The horizontal width of each image is $\sim 50 \mathrm{~m}$. The images show a lake in sunlight (A), a central deciduous wooded area (B) and an isolated building (labelled C).

Figure 9. Altitude estimates obtained across the case study profile. Images of the 3 zones (A, B and C) are shown in Figure 8. The four estimates of altitude shown are Radar (Ralt at $4 \mathrm{~Hz}$ ), raw Laser at $200 \mathrm{~Hz}$, Lmax processed Laser at $4 \mathrm{~Hz}$. The height above geoid data (GPS-z) has had a constant value removed to allow inclusion in the plot. 
Figure 10. 1D inversion of the 4 frequency data across the case study profile. The number of layers is assumed unknown and a multi- layer smooth (Occam) inversion is used. The arrows denote the width of the canopy zone. The filled rectangle denotes the zone of maximum height of canopy. (a) Results obtained with radar altimeter, (b) Results obtained using Lmax processed laser altimeter data.

Figure 11. 1D inversion of the 4 frequency data across the case study profile. The number of layers is assumed known and a 5-layer laterally-constrained inversion is used. The arrows denote the width of the canopy zone. The filled rectangle denotes the zone of maximum height of canopy. (a) Results obtained with radar altimeter, (b) Results obtained using Lmax processed laser altimeter data.

Figure 12. Topographic map across a portion of a detailed geophysical survey in southern Finland. Flight line spacing is $75 \mathrm{~m}$. The area contains 4 water bodies shown in blue. The red symbols denote locations along flight lines where processed (Lmax) laser null returns were observed. Topographic Map (C) National Land Survey of Finland, permission no. 13/MML/08. 


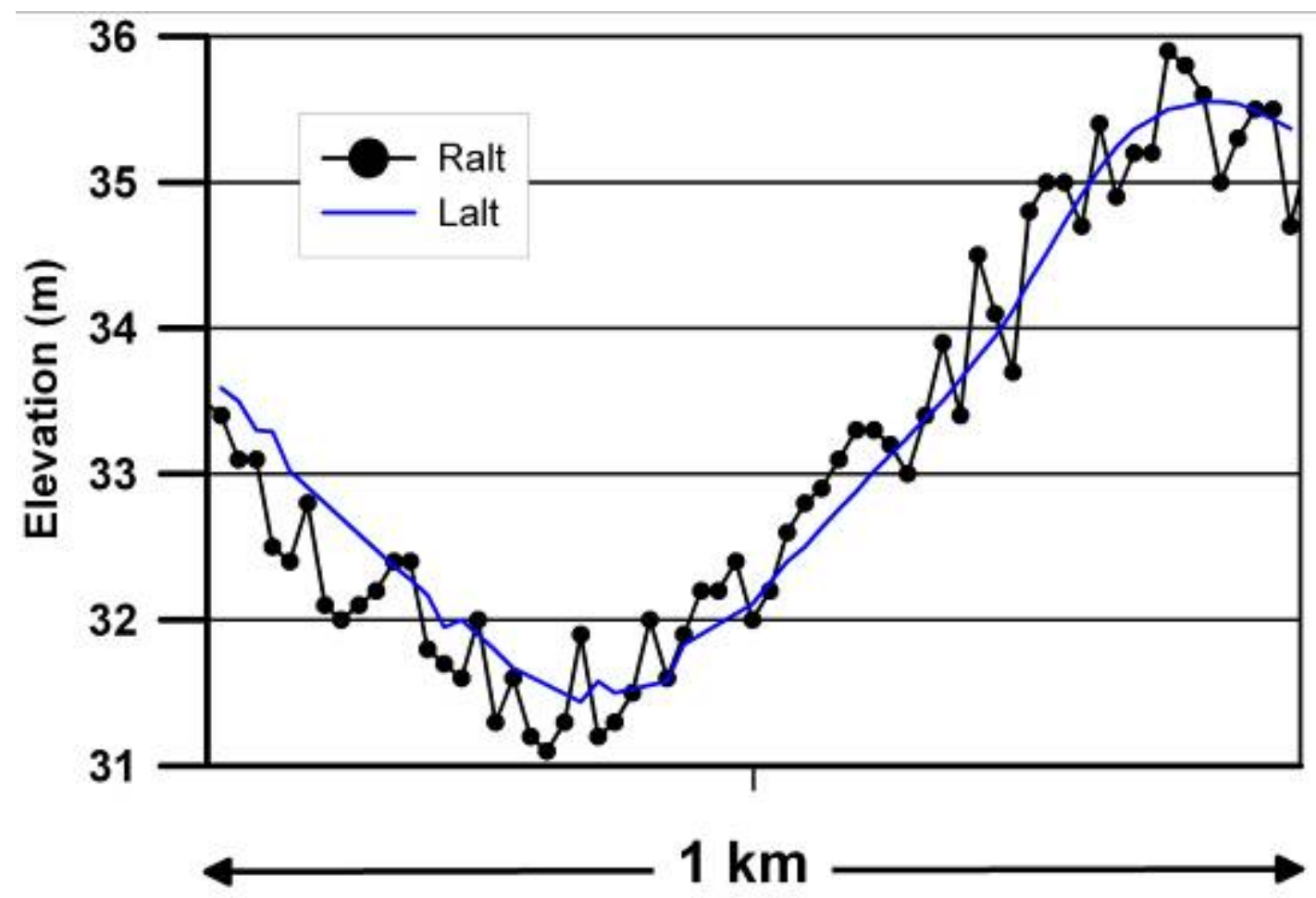

Figure 1. Example of Radar (Ralt) and Laser (Lalt) altimeter data recorded over a 1 $\mathrm{km}$ section of a lake. The sampling interval is $4 \mathrm{~Hz}$. The aircraft height undulates by 4 $\mathrm{m}$ across the $1 \mathrm{~km}$ profile. 


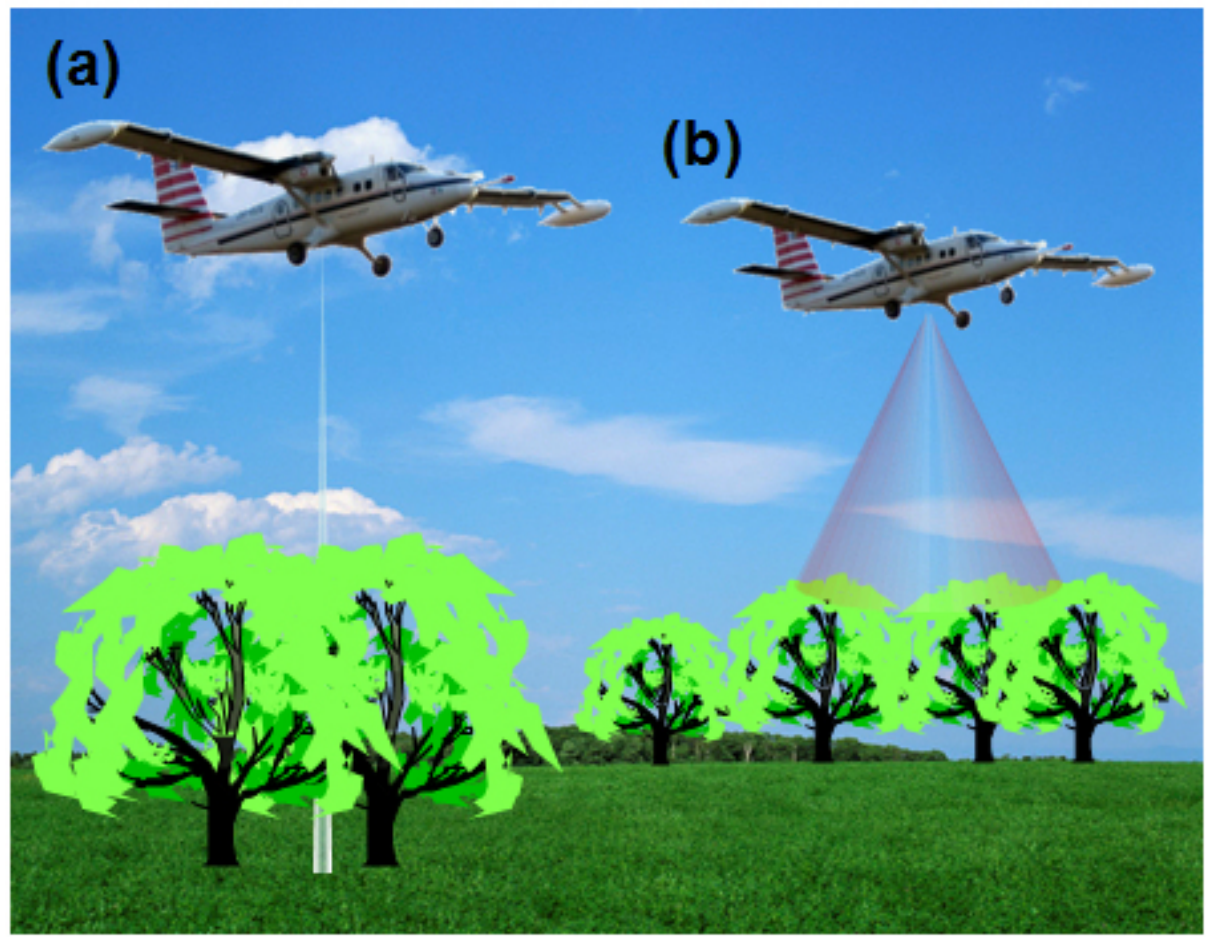

Figure 2. Schematic representation of beamwidths associated with (a) a laser altimeter and (b) a radar altimeter. 


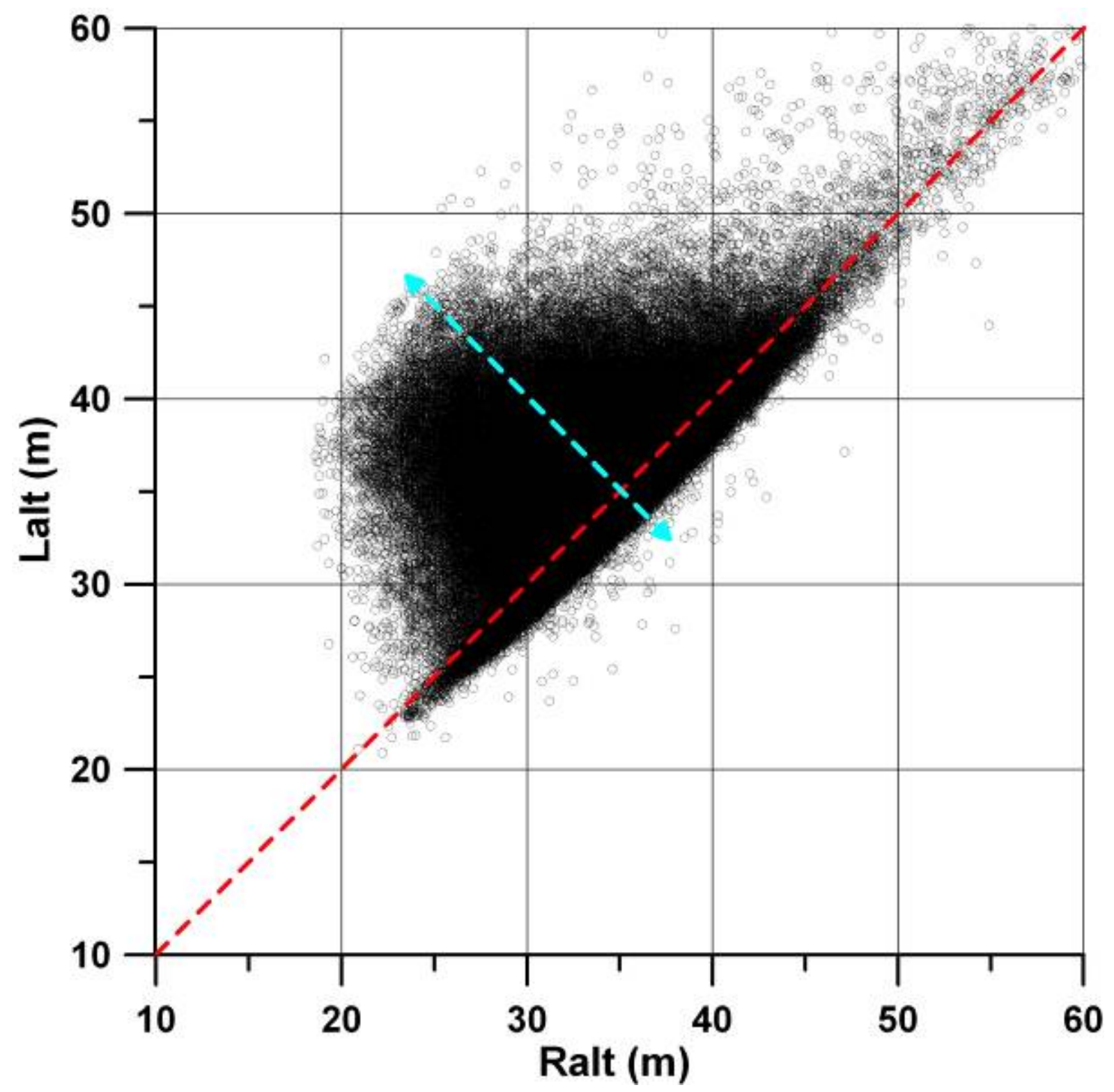

Figure 3. Example of typical behaviour observed between Radar altitude (Ralt) and Laser (Lalt) altimeter data in a largely rural survey area. The Lalt data have been processed by the Lmax algorithm described in the text. The data sampling interval is 4 $\mathrm{Hz}$ and the plot contains $\sim 150,000$ data points although the plot has been restricted to a maximum height of $60 \mathrm{~m}$. The double-arrowed line indicates a scale of $20 \mathrm{~m}$. 


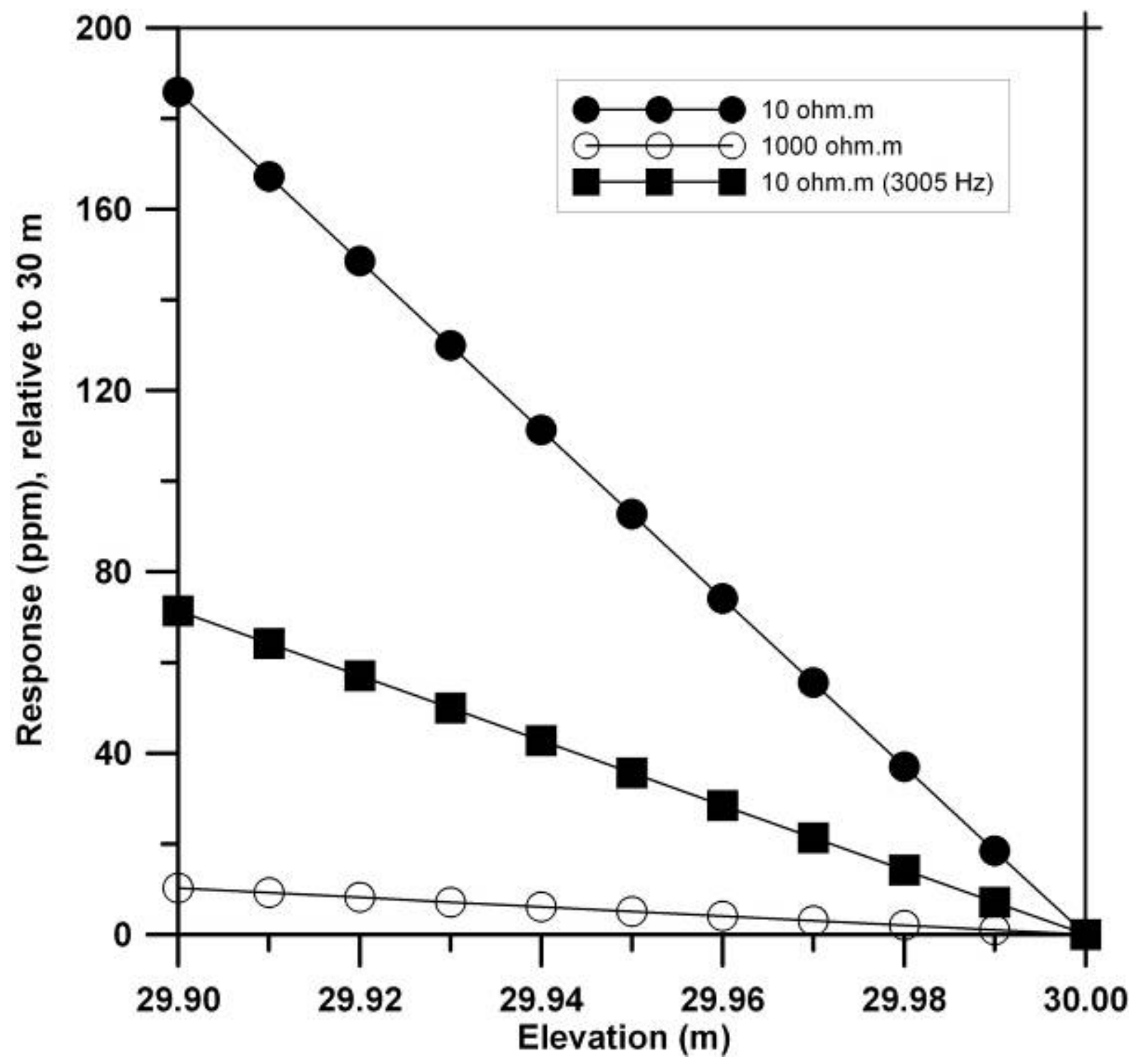

Figure 4. Altitude dependence of coupling ratios across an elevation range of $10 \mathrm{~cm}$. Responses are relative and normalised to those at an altitude of $30 \mathrm{~m}$. The highest frequency of the AEM-05 system $(24510 \mathrm{~Hz})$ is shown for 2 half-space resistivity values (10 and $1000 \mathrm{ohm} . \mathrm{m})$. The response at a lower frequency of $3005 \mathrm{~Hz}$ for a 10 ohm.m half-space is also shown for reference. 


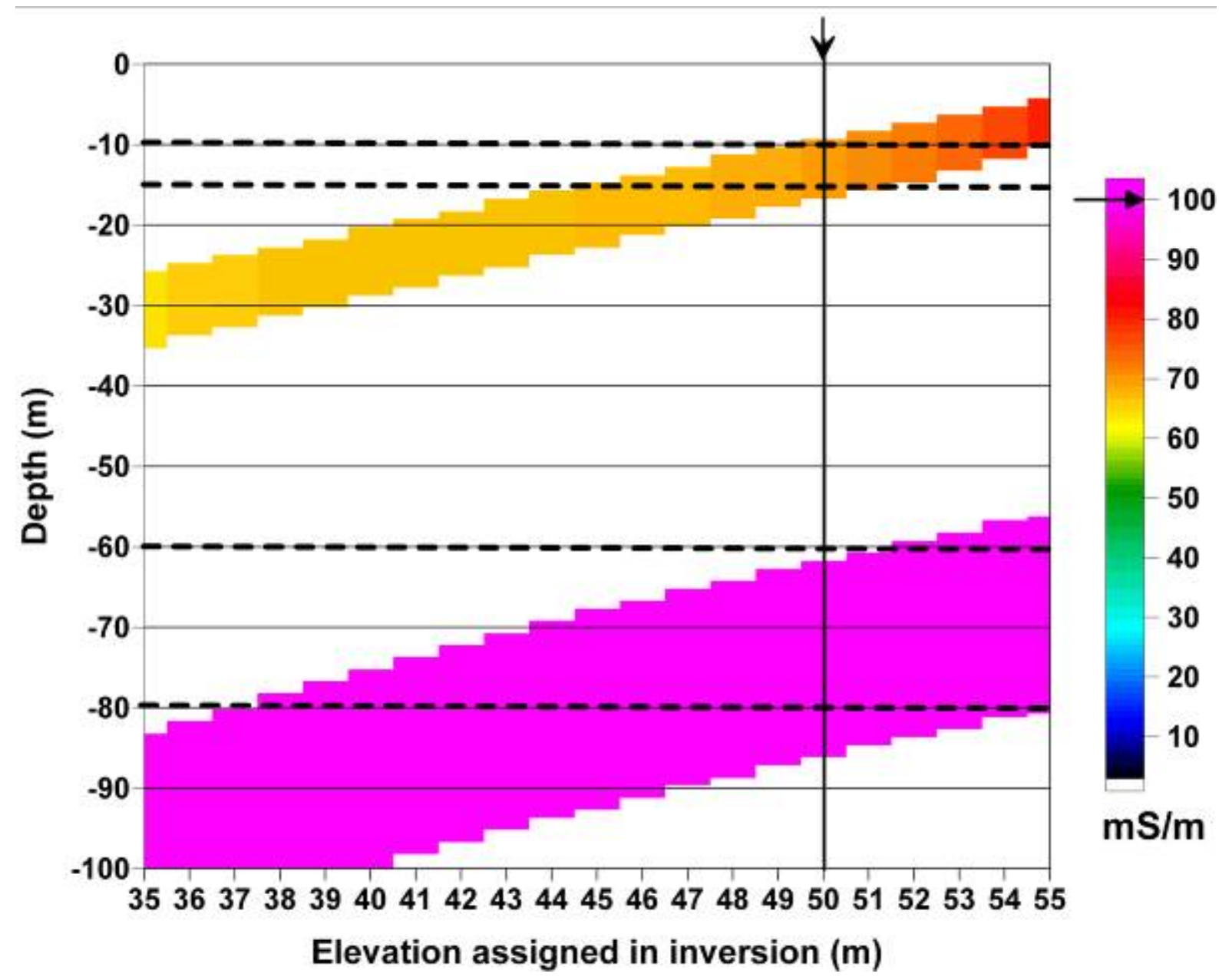

Figure 5. 1D inversion of synthetic model results illustrating the effect of incorrect altitude assignment on the detection of two conductive zones. The number of layers (4 above a half-space) is assumed known and a few layer inversion is used. The data are correct at an altitude of $50 \mathrm{~m}$. The results for a series of altitude errors between $-15 \mathrm{~m}$ (underestimated) and $+5 \mathrm{~m}$ (overestimated) are shown. The depths/thicknesses of the 2 conductive zones $(100 \mathrm{mS} / \mathrm{m})$ are indicated by dash lines. 


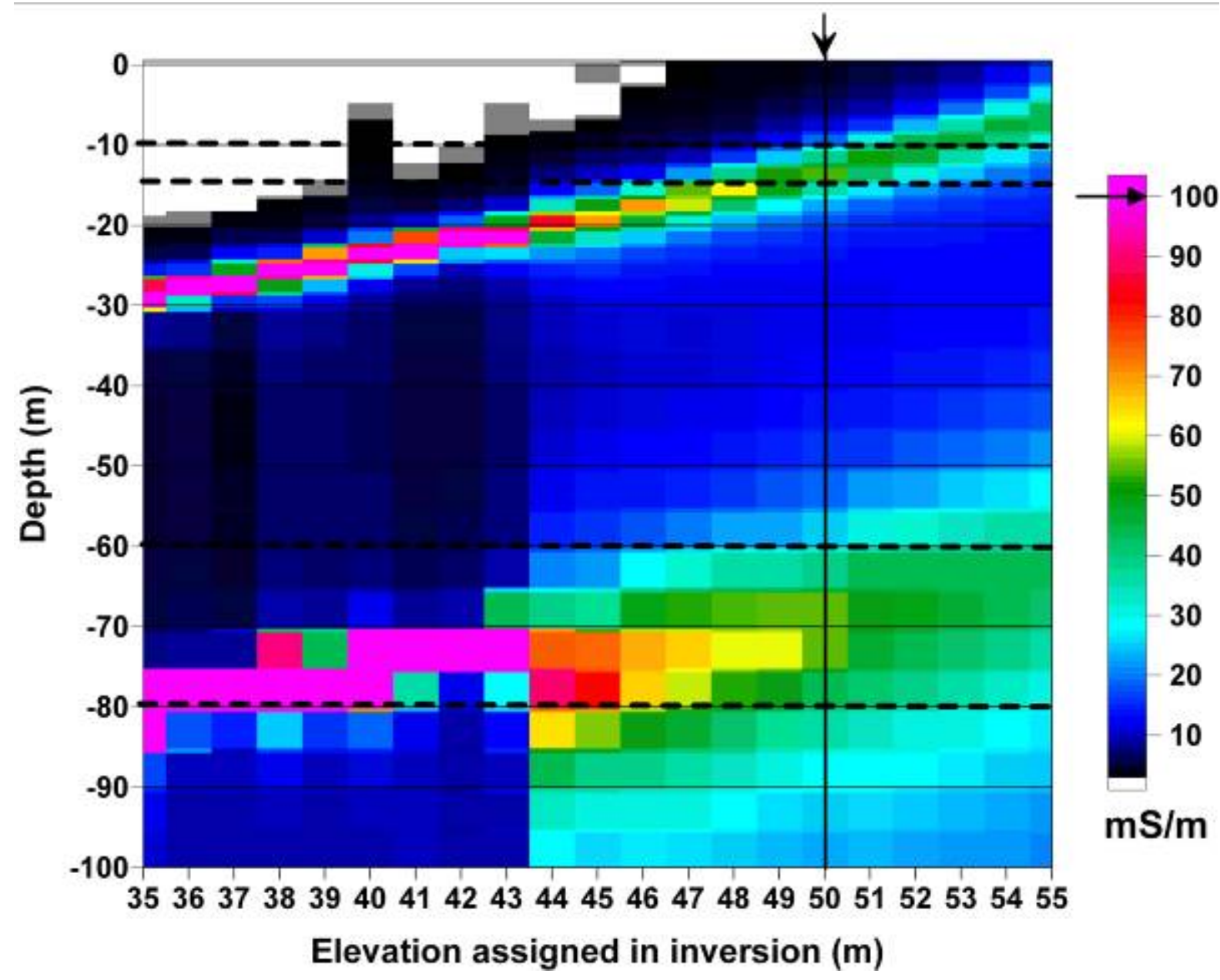

Figure 6. 1D inversion of synthetic model results illustrating the effect of incorrect altitude assignment on the detection of two conductive zones. The number of layers is assumed unknown and a multi-layer smooth (Occam) inversion is used. The data are correct at an altitude of $50 \mathrm{~m}$. The results for a series of altitude errors between $-15 \mathrm{~m}$ (underestimated) and $+5 \mathrm{~m}$ (overestimated) are shown. The depths/thicknesses of the 2 conductive zones $(100 \mathrm{mS} / \mathrm{m})$ are indicated by dash lines. 


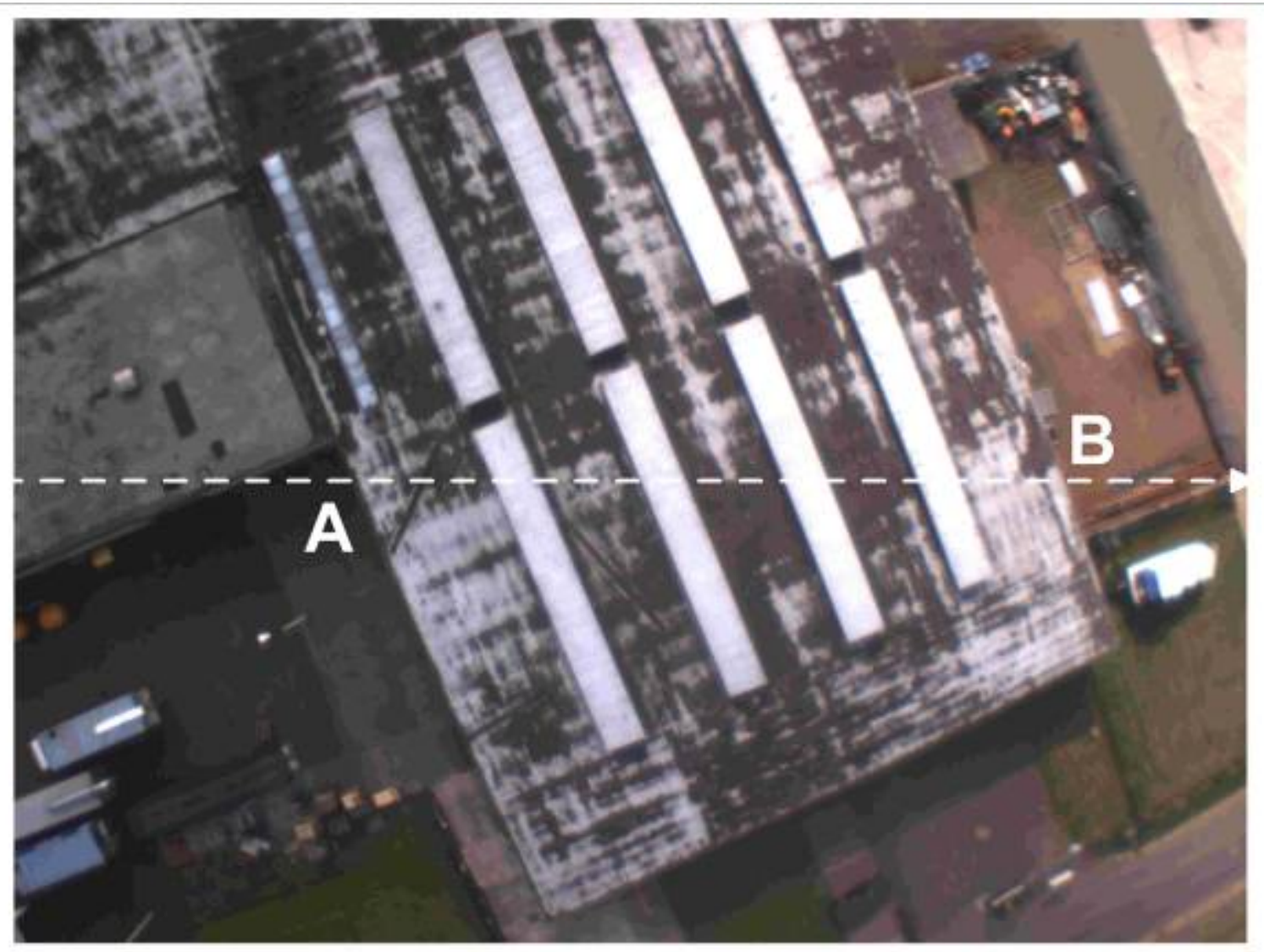

Figure 7a. Image obtained from downward-looking video above an industrial unit. The flight path is shown by the horizontal dash line. The distance across the edges of the unit $(\mathrm{A}, \mathrm{B})$ is $40 \mathrm{~m}$. The survey elevation is $\sim 66 \mathrm{~m}$. 


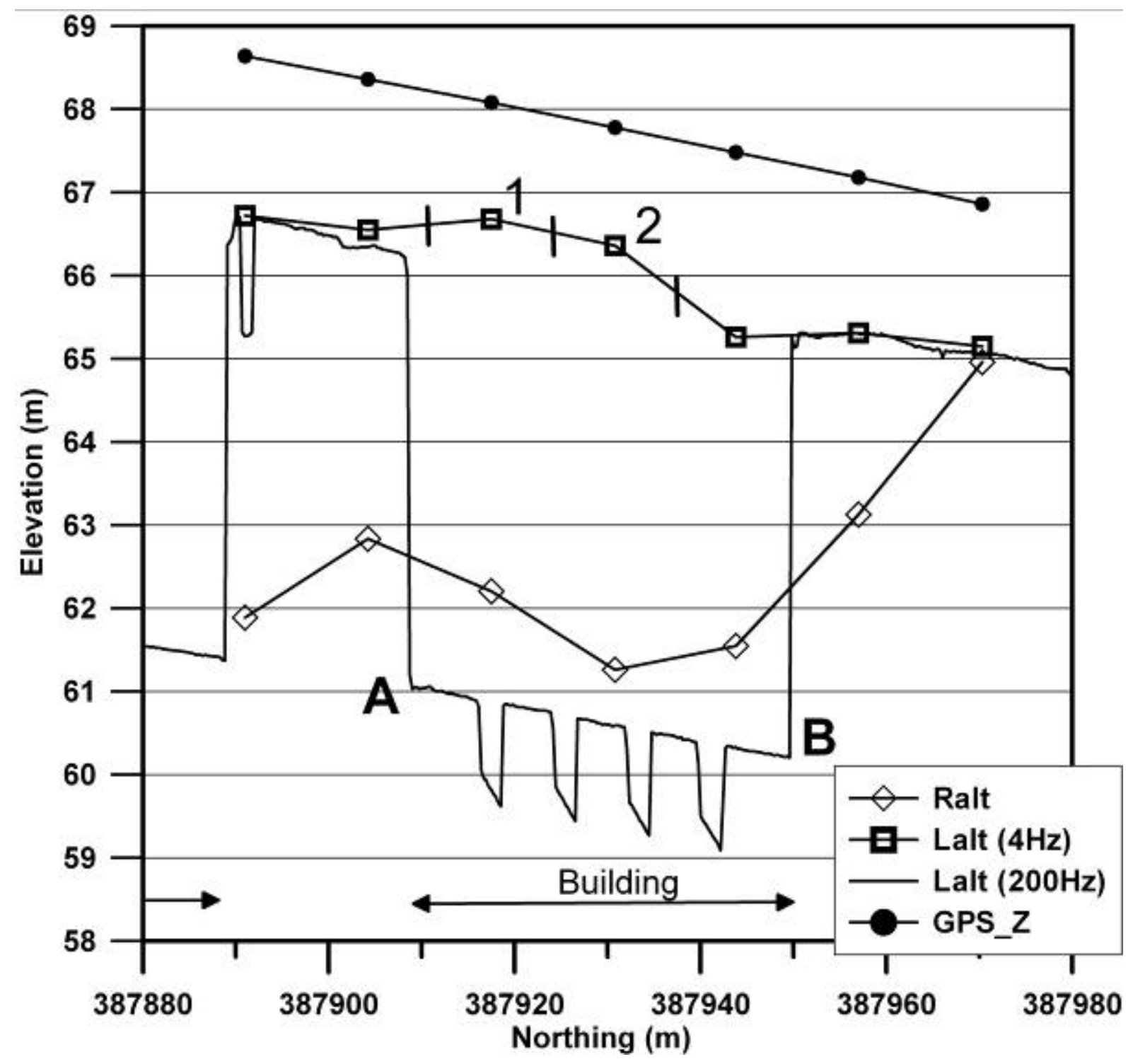

Figure 7b. Altitude estimates obtained across the industrial unit shown in Figure 7a. The main building is indicated by the arrowed line A,B, a second building is also indicated. The four estimates of altitude shown are Radar (Ralt at $4 \mathrm{~Hz}$ ), raw Laser at $200 \mathrm{~Hz}$, Lmax processed Laser at $4 \mathrm{~Hz}$. The height above geoid data (GPS-z) has had a constant value removed to allow inclusion in the plot. 

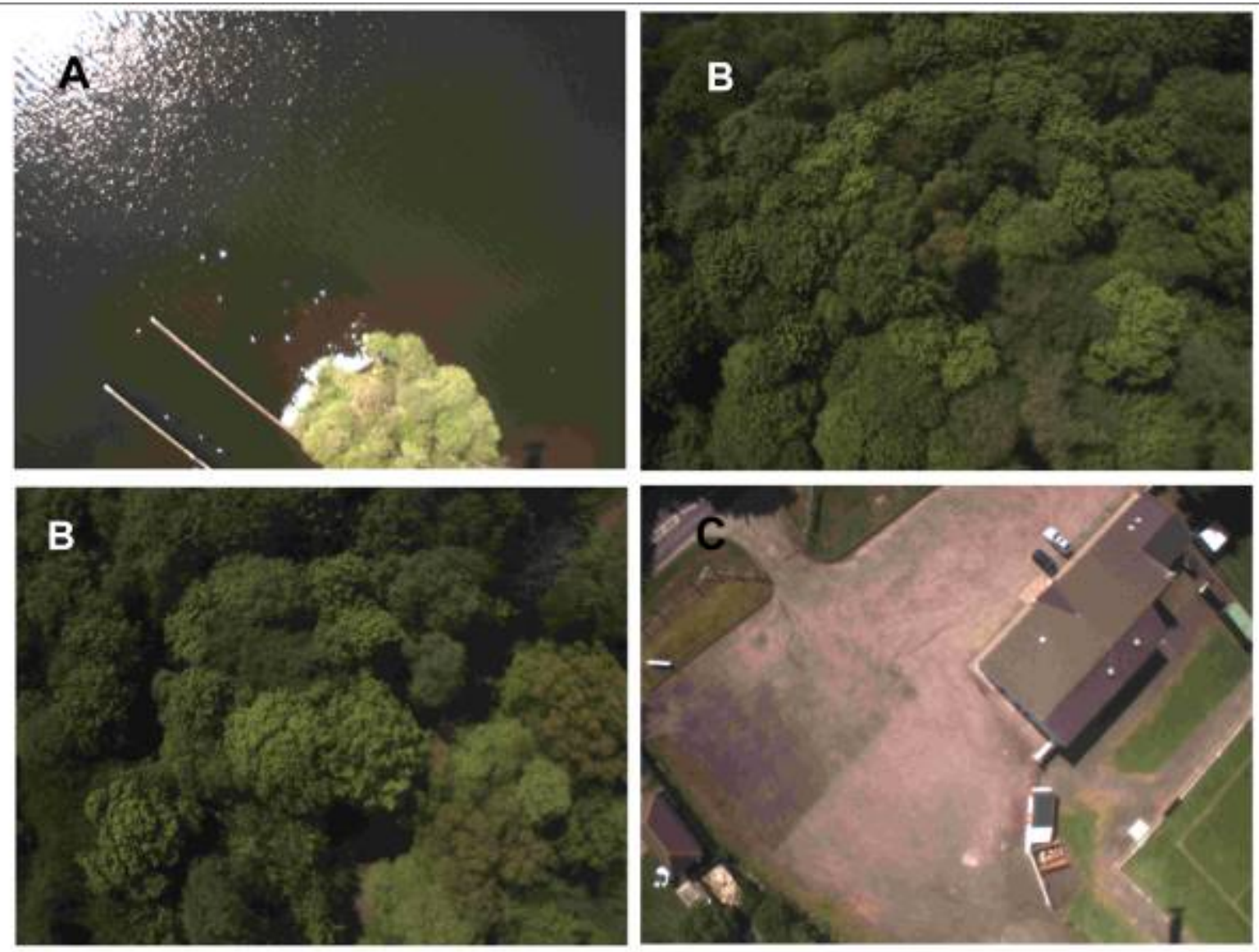

Figure 8. Four images obtained from the downward-looking video across the case study flight line. The horizontal width of each image is $\sim 50 \mathrm{~m}$. The images show a lake in sunlight (A), a central deciduous wooded area (B) and an isolated building (labelled C). 


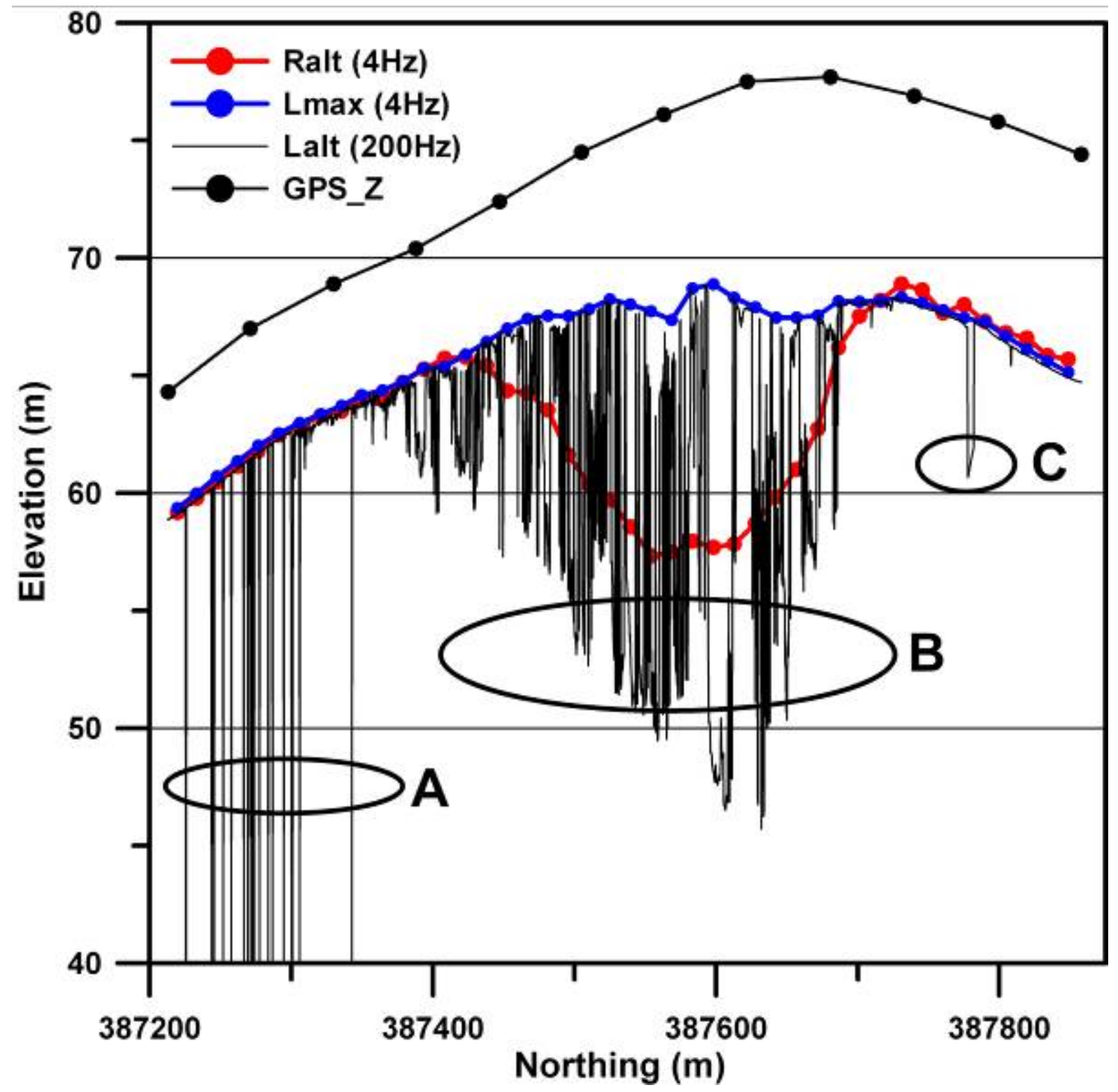

Figure 9. Altitude estimates obtained across the case study profile. Images of the 3 zones (A, B and C) are shown in Figure 8. The four estimates of altitude shown are Radar (Ralt at $4 \mathrm{~Hz}$ ), raw Laser at $200 \mathrm{~Hz}$, Lmax processed Laser at $4 \mathrm{~Hz}$. The height above geoid data (GPS-z) has had a constant value removed to allow inclusion in the plot. 


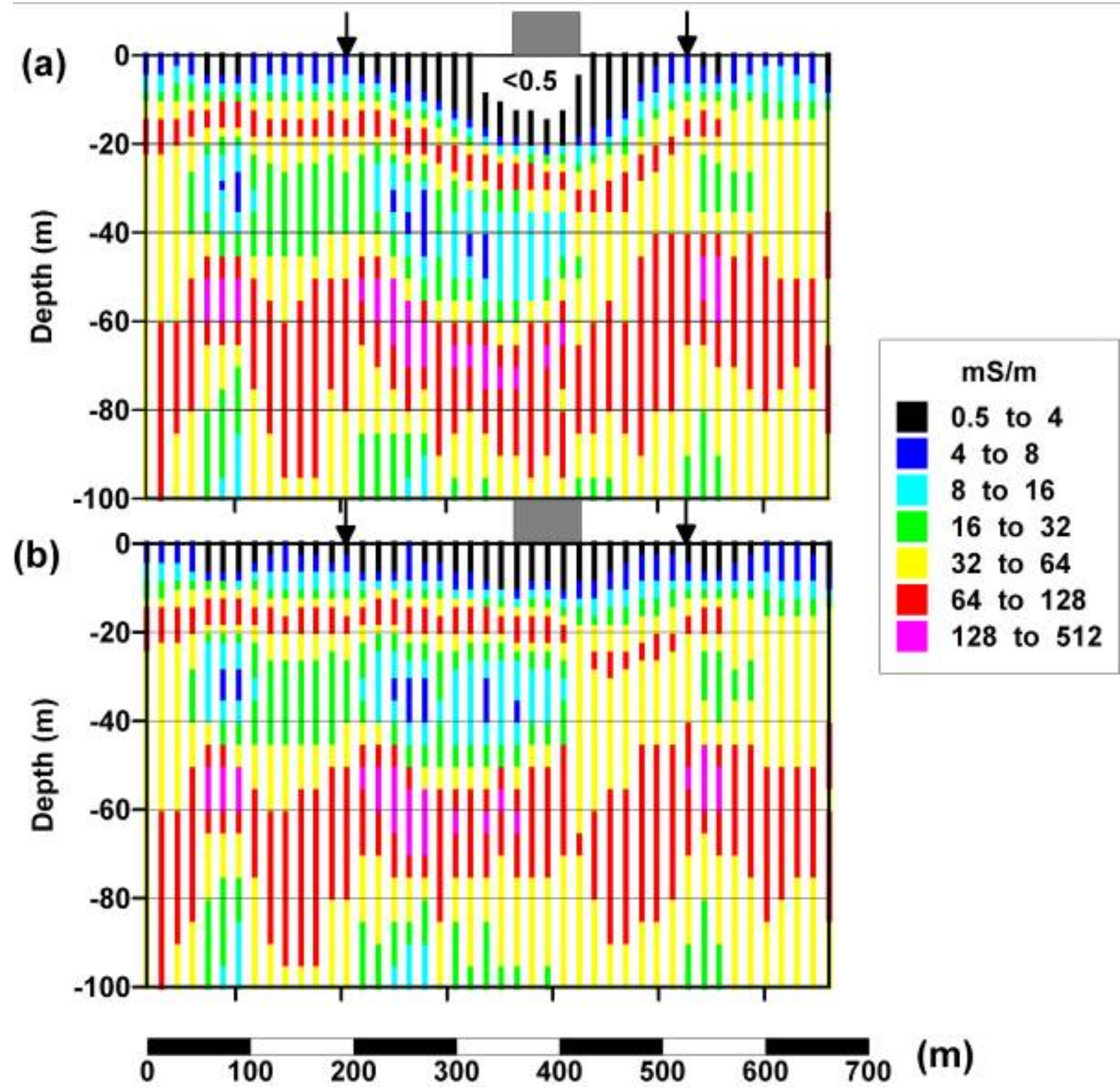

Figure 10. 1D inversion of the 4 frequency data across the case study profile. The number of layers is assumed unknown and a multi- layer smooth (Occam) inversion is used. The arrows denote the width of the canopy zone. The filled rectangle denotes the zone of maximum height of canopy. (a) Results obtained with radar altimeter, (b) Results obtained using Lmax processed laser altimeter data. 


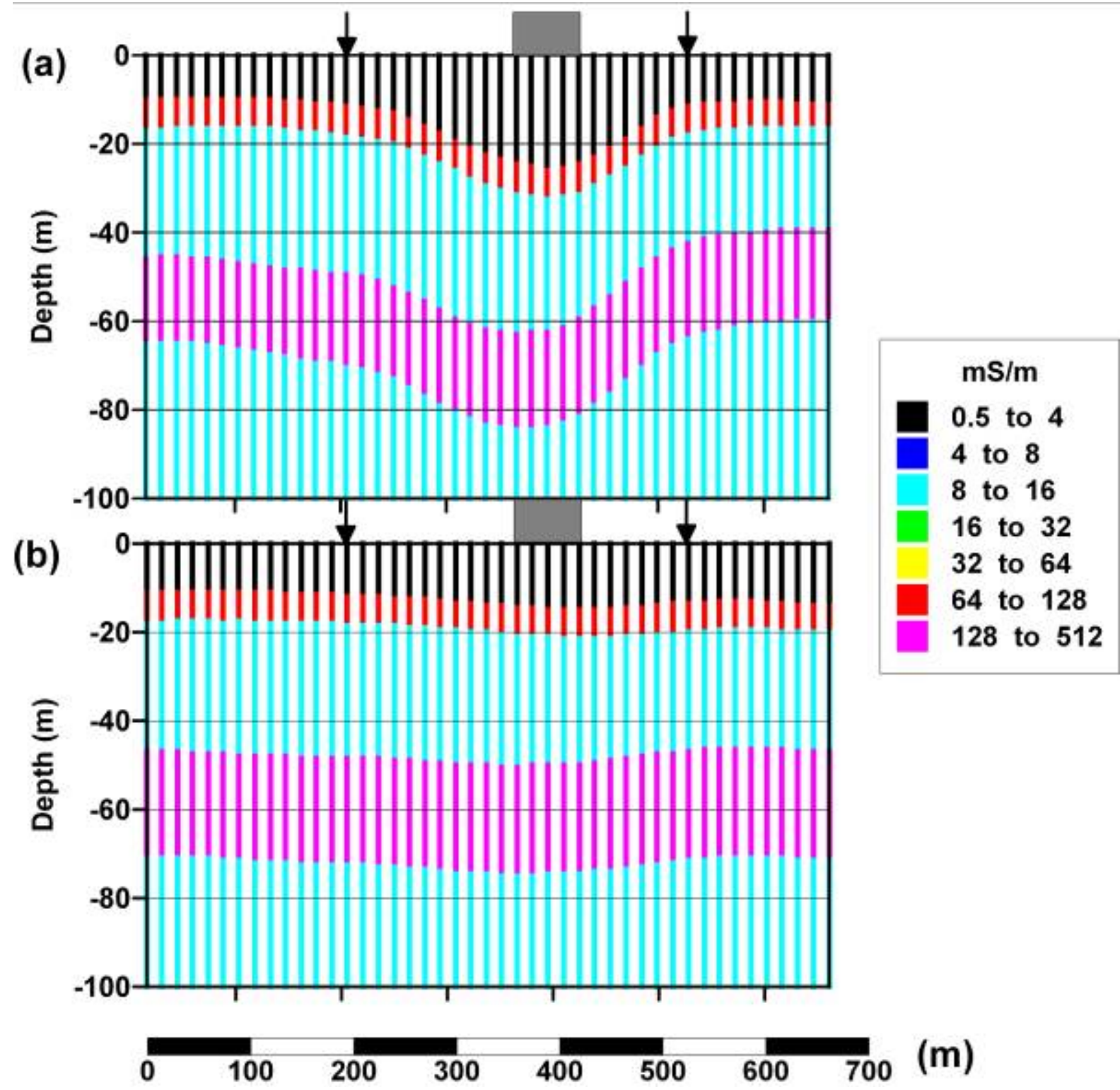

Figure 11. 1D inversion of the 4 frequency data across the case study profile. The number of layers is assumed known and a 5-layer laterally-constrained inversion is used. The arrows denote the width of the canopy zone. The filled rectangle denotes the zone of maximum height of canopy. (a) Results obtained with radar altimeter, (b) Results obtained using Lmax processed laser altimeter data. 


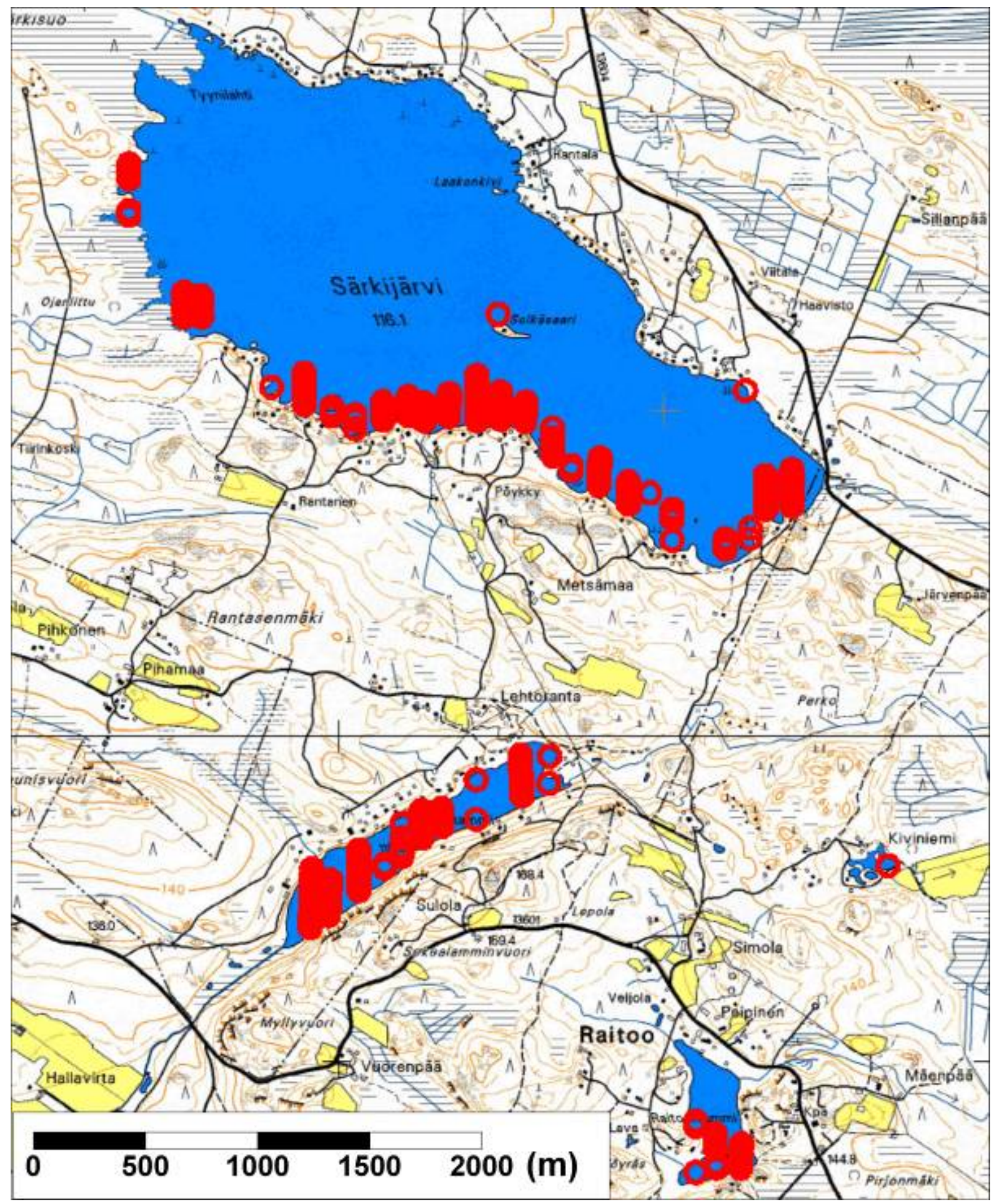

Figure 12. Topographic map across a portion of a detailed geophysical survey in southern Finland. Flight line spacing is $75 \mathrm{~m}$. The area contains 4 water bodies shown in blue. The red symbols denote locations along flight lines where processed (Lmax) laser null returns were observed. Topographic Map (C) National Land Survey of Finland, permission no. 13/MML/08. 